\title{
Epidemiology and Mortality-Associated Factors of Invasive Fungal Disease in Elderly Patients: A 20-Year Retrospective Study from Southern China
}

This article was published in the following Dove Press journal:

Infection and Drug Resistance

\author{
Yingying Gong ${ }^{1, *}$ \\ Chen $\mathrm{Li}^{\mathrm{l}}$ ** \\ Cuicui Wang ${ }^{2, *}$ \\ Jin $\mathrm{Li}^{\prime}$ \\ Meilin Ding' \\ Dongying Chen (iD) ${ }^{2}$ \\ Minxi Lao (iD) 1,2 \\ 'Department of Geriatrics, The First \\ Affiliated Hospital of Sun Yat-sen \\ University, Guangzhou, People's Republic \\ of China; ${ }^{2}$ Department of Rheumatology, \\ The First Affiliated Hospital of Sun Yat- \\ sen University, Guangzhou, People's \\ Republic of China
}

*These authors contributed equally to this work

\begin{abstract}
Introduction: Invasive fungal disease (IFD) is a life-threatening infection. The epidemiology and clinical features of IFD in the elderly population are less discussed. The aim of this study was to explore the epidemiology and mortality-associated factors for IFD in the elderly inpatients.
\end{abstract}

Methods: A retrospective study enrolling 512 elderly inpatients from The First Affiliated Hospital of Sun Yat-sen University during the last two decades was performed.

Results: The annual prevalence of IFD was $0.1-0.5 \%$. Candidiasis was the most common $(236 / 521,45.3 \%)$. An increasing trend was observed in aspergillosis from $11.1 \%$ in year 1998 to $28.8 \%$ in year 2018 . The common infective sites of candidiasis were abdominal cavity $(83 / 236,35.2 \%)$ and bloodstream $(55 / 236,23.3 \%)$. Invasive aspergillosis mainly developed in the sinus $(74 / 149,49.7 \%)$ and lung $(65 / 149,43.6 \%)$. Patients with diabetes mellitus (DM) $(59 / 126,46.8 \%)$, solid organ malignancy $(84 / 114,73.7 \%)$, chronic kidney disease (CKD) $(40 / 62,64.5 \%)$ or receiving operation $(109 / 147,74.1 \%)$ were prone to develop candidiasis, while aspergillosis was usually complicated in patients with chronic obstructive pulmonary disease (COPD) $(25 / 51,49.0 \%)$. The all-cause mortality rate was $25.9 \%(135 / 521)$, and patients aged $\geq 80$ years were the riskiest $(20 / 51,39.2 \%)$. Lymphopenia $(59.5 \%$ vs $17.3 \%, P<0.001)$ was significant in deceased patients with mold infection. Higher proportion of non-survivors with invasive candidiasis received central venous catheterization $(\mathrm{CVC})(68.4 \%$ vs $40.6 \%, P<0.001)$ or indwelling urinary catheter (68.4\% vs $46.3 \%, P=0.001)$.

Conclusion: IFD is a life-threatening complication especially in the oldest-old. Surveillance on lymphopenia, prompt treatment and reduce invasive procedures could benefit the prognosis.

Keywords: invasive fungal disease, elderly, mortality, hypoalbuminemia

\section{Introduction}

Department of Geriatrics, The First Affiliated Hospital of Sun Yat-Sen

University, No. 58, Zhongshan 2nd Road, Guangzhou 510080, People's Republic of China

Email laomx3@mail.sysu.edu.cn

Dongying Chen

Department of Rheumatology, The First Affiliated Hospital of Sun Yat-sen

University, No. 58, Zhongshan 2nd Road, Guangzhou 510080, People's Republic of China

Email cdongying@163.com
Elderly people present many physical features different from their younger counterparts. Frail individuals, often complicating with underlying chronic diseases, have limited reserves to withstand health stressors. One of the noticeable disadvantages ing susceptibility to infection. It was reported that up to 1 in 10 individuals in the long-term care has experienced infection. ${ }^{1}$

Invasive fungal disease (IFD) is life-threatening infection. The estimates are mainly derived from the immunocompromised population, with scant data focusing in the elderly population is the weakening barriers to pathogens, leading to increas- 
on older adults. The elderly people are potential immunodeficient hosts. Several immune alterations have been noticed in the elderly with cellular and humoral immunity being impaired. ${ }^{2}$ Except for the established risk factors, the high rate of comorbidity, malnutrition, and polypharmacy, which are common in the older adults, could intervene the clinical management of IFD. Aging is related to the occurrence of IFD such as intraabdominal candidiasis (IAC) and candidemia. ${ }^{3,4}$ The mortality rate of IFD in patients aged $\geq 65$ increases over time, ${ }^{5}$ and was reported to be $33-44 \%$ in elderly patients. ${ }^{6,7}$ However, until recently, few studies were designed to explore IFD in Chinese elderly population, and the IFD-related mortality remains to be clarified.

Herein, we leveraged a 20 -year retrospective study, aiming to determine the epidemiology and associated factors of mortality in elderly patients with IFD from Southern China.

\section{Methods}

\section{Study Design}

A retrospective study was performed on the inpatients with age $\geq 60$ years, the age beyond which was categorized as elderly according to the Chinese law, from the First Affiliated Hospital of Sun Yat-Sen University from January 1st, 1998 to December 31st, 2018. The International Classification of Diseases-10 (ICD-10) coding of discharged diagnoses was used to identify patients with IFD. Records were re-evaluated by two geriatricians (Yingying Gong, Minxi Lao). One episode of IFD from each patient was collected. Patients without complete data or developing IFD at the age $<60$ were excluded. This work was conducted according to the provisions of the Declaration of Helsinki.

\section{Case Definition}

The diagnostic criteria for IFD are shown in Table 1. IFD was designated as proven, probable or possible according to the EORTC/MSG Guidelines, ${ }^{8,9}$ and Chinese expert consensus on candidiasis. ${ }^{10}$ Fungal infection involving skin, genitalia, and oral cavity was excluded. Disseminated IFD was defined in the case of fungal septicemia, or IFD involving two or more non-consecutive organs.

\section{Demographical and Clinical Data}

Demographic and clinical data were collected from the medical records. Clinical characteristics of IFD included symptoms and signs, sites of infection, and anti-fungi treatment. Underlying diseases were recorded. Chronic kidney disease (CKD) is defined as a progressive loss of renal function measured by GFR $<60 \mathrm{~mL} / \mathrm{min}$ per $1.73 \mathrm{~m}^{2}$, or markers of kidney damage, or both, lasting for more than 3 months. ${ }^{11}$ Hypertension is defined as systolic blood pressure $\geq 140 \mathrm{mmHg}$ and/or diastolic blood pressure $\geq 90 \mathrm{mmHg}{ }^{12}$ Coronary artery disease (CAD) is a stage of atherosclerosis that involves fatty deposits inside the coronary arterial walls, leading to narrowing of the arteries, and causing clinical symptoms such as angina or heart attack. Chronic obstructive pulmonary disease (COPD) is defined as persistent respiratory symptoms and airflow limitation (presented as postbronchodilator forced expiratory volume in $1 \mathrm{~s}$ (FEV1)/ forced vital capacity (FVC) ratio of less than 0.70 ) which is due to airway and/or alveolar abnormalities, usually caused by significant exposure to noxious particles or gases. ${ }^{13,14}$ Diabetes mellitus (DM) was defined as having any one of the following: 1) fasting plasma glucose level $\geq 7 \mathrm{mmol} / 1$ (126 mg/dl), or 2) plasma glucose $\geq 11.1 \mathrm{mmol} / 1$ (200 mg/ dl) $2 \mathrm{hrs}$ after a 75 -g oral glucose load as in a glucose tolerance test (OGTT), or 3) symptoms of high blood glucose and casual plasma glucose $\geq 11.1 \mathrm{mmol} / 1(200 \mathrm{mg} / \mathrm{dl}){ }^{15}$ Connective tissue disease (CTD) is a group of diseases that mainly affect the connective tissue, caused by autoimmune disorders. Laboratory data included routine blood tests and blood biochemistry. Neutropenia was defined as an absolute neutrophil count (ANC) of less than $2.0 \times 10^{9} / \mathrm{L}$ and graded as: grade I (ANC $\left.1.5-1.9 \times 10^{9} / \mathrm{L}\right)$, grade II (ANC $1.0-1.4 \times 10^{9} / \mathrm{L}$ ), grade III $\left(\right.$ ANC $0.5-0.9 \times 10^{9} / \mathrm{L}$ ) or grade IV $\left(\mathrm{ANC}<0.5 \times 10^{9} / \mathrm{L}\right) .{ }^{19}$ Lymphopenia was defined as peripheral blood lymphocyte count $<1.0 \times 10^{9} / \mathrm{L}$. Anemia was defined as hemoglobin $<120 \mathrm{~g} / \mathrm{L}$ in males and hemoglobin $<110 \mathrm{~g} / \mathrm{L}$ in females at sea level. The severity of anemia was classified as: mild ( $>90 \mathrm{~g} / \mathrm{L})$, moderate $(60-90 \mathrm{~g} / \mathrm{L})$, severe $(30-59 \mathrm{~g} / \mathrm{L})$ or very severe $(<30 \mathrm{~g} / \mathrm{L}) .{ }^{20}$ Hypoalbuminemia was defined as serum albumin level $<3.5 \mathrm{~g} / \mathrm{dl}$. Charlson comorbidity score was calculated according to previous report. ${ }^{16}$ Microbial culture and biopsy findings were recorded if available. Polypharmacy was defined as receiving $>10$ medications at the time of IFD onset. Prolonged hospitalization was defined as hospital stay longer than 10 days. Prolonged use of glucocorticoids (GC) was defined as a mean minimum dose of $0.3 \mathrm{mg} / \mathrm{kg} /$ day of prednisone equivalent for $>3$ weeks. Potential-associated factors within 3 months prior to IFD onset included operation, the use of broad-spectrum antibiotics, central venous catheter (CVC), indwelling urinary catheter, and immunosuppressive therapy. Initial 
Table I Criteria for Diagnosis of IFD in the Elderly Patients

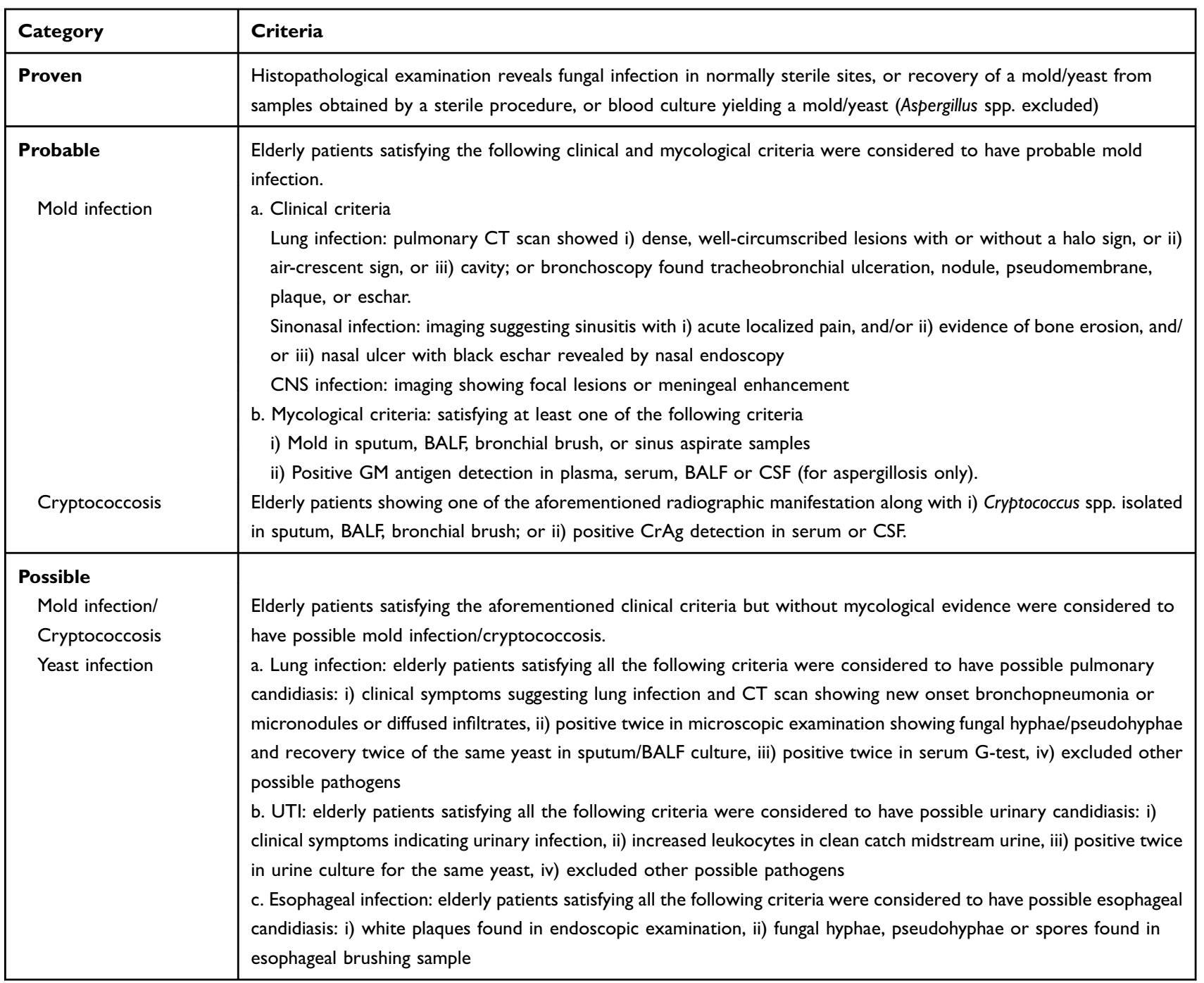

Abbreviations: BALF, bronchoalveolar lavage fluid; CNS, central nervous system; CrAg, cryptococcal capsule polyglycan antigen; CSF, cerebrospinal fluid; CT, computed tomography; G-test, $\beta$-D-glucan detection; GM, galactomannan; IFD, invasive fungal disease; UTI, urinary tract infection.

antifungal treatment was considered adequate if the infecting fungi were ultimately shown to be susceptible and the dosage of antifungal used was complied with the recommendation ${ }^{10,17}$ within the first $24 \mathrm{~h}$ from culture positivity. Surgical resection alone was considered adequate to treat Aspergillus fungal ball of the paranasal sinus. ${ }^{18}$

\section{Statistical Analysis}

Normally distributed quantitative variables were expressed as mean $\pm \mathrm{SD}$. Qualitative variables were expressed as absolute numbers and percentages. Proportions were compared using Fisher's exact test or Pearson $\chi^{2}$ test. Betweengroup comparison was evaluated using Student's $t$-test, or one-way ANOVA for continuous variables with normal distribution. Odds ratio (OR) and corresponding 95\% confidence interval (CI) of clinically significant variables with a $P<0.1$ in univariate regression analysis was adjusted by multivariate logistic regression analysis, applying forward procedure. The two-sided level of significance was set at $P<0.05$. All statistical analysis was performed with the SPSS 19 statistical package (IBM).

\section{Results}

\section{Demographics}

A total of 189,843 patients were screened, and 651 potential patients were selected. Ninety-four patients were excluded for not meeting the inclusion criteria and 36 were excluded for data incompleteness. Finally, 521 patients (323 males, 198 females) with IFD were included. Except one patient was Yemenese, the rest of the 520 
patients were Han nationality. The mean (SD) age was 69.6 \pm 7.1 years (range, 60-92). Proven IFD accounted for $71.2 \%(371 / 521)$ of the cases, probable cases for $16.1 \%$
(84/521), and possible cases for 12.7\% (66/521). Baseline characteristics are shown in Table 2 . Two $(0.4 \%)$ patients were infected with human immunodeficiency virus (HIV).

Table 2 Participants Characteristics According to Age

\begin{tabular}{|c|c|c|c|c|}
\hline & \multicolumn{3}{|l|}{ Age, Years } & \multirow[t]{2}{*}{$P$ value } \\
\hline & $60-69(n=272)$ & $70-79(n=198)$ & $\geq 80(n=5 I)$ & \\
\hline \multicolumn{5}{|l|}{ Baseline Characteristics } \\
\hline Male:Female & $162: 110$ & $126: 72$ & $35: 16$ & 0.39 \\
\hline $\mathrm{BMI}, \mathrm{kg} / \mathrm{m}^{2}$, mean $\pm \mathrm{SD}$ & $22.9 \pm 3.5$ & $22.4 \pm 3.4$ & $22.3 \pm 3.0$ & 0.22 \\
\hline Tobacco use, n (\%) & $58(21.3)$ & $43(21.7)$ & $17(33.3)$ & 0.16 \\
\hline Charlson score, mean $\pm S D$ & $3.7 \pm 2.0$ & $5.0 \pm 2.0$ & $5.2 \pm 2.0$ & $<0.001 *$ \\
\hline Polypharmacy, n (\%) & $47(17.3)$ & $36(18.2)$ & II (2I.6) & 0.76 \\
\hline \multicolumn{5}{|l|}{ Comorbidities } \\
\hline Hypertension, n (\%) & $94(34.6)$ & $91(46.0)$ & $22(43.1)$ & $0.04 *$ \\
\hline Hematological or solid organ malignancy, n (\%) & $62(22.8)$ & $53(26.8)$ & $15(29.4)$ & 0.48 \\
\hline DM, n (\%) & $55(20.2)$ & $62(31.3)$ & $10(19.6)$ & $0.01 *$ \\
\hline CKD, n (\%) & $30(11.0)$ & $24(12.1)$ & $8(15.7)$ & 0.64 \\
\hline CTD, n (\%) & $25(9.2)$ & $4(2.0)$ & $\mathrm{I}(2.0)$ & $0.002 *$ \\
\hline CAD, n (\%) & $18(6.6)$ & $30(15.2)$ & $10(19.6)$ & $0.002 *$ \\
\hline COPD, n (\%) & II (4.0) & $30(15.2)$ & $10(19.6)$ & $<0.001 *$ \\
\hline Solid organ transplantation, $\mathrm{n}(\%)$ & $8(2.9)$ & $2(1.0)$ & $0(0)$ & 0.19 \\
\hline Acute stroke, n (\%) & $6(2.2)$ & $7(3.5)$ & $\mathrm{I}(2.0)$ & 0.64 \\
\hline \multicolumn{5}{|l|}{ Associated Factors } \\
\hline Prolonged hospitalization, n (\%) & $29(10.7)$ & $23(11.6)$ & $10(19.6)$ & 0.19 \\
\hline Recent operation, n (\%) & $72(26.5)$ & $59(29.8)$ & $16(31.4)$ & 0.69 \\
\hline ICU admission, $n(\%)$ & $81(29.8)$ & $90(45.5)$ & $32(62.7)$ & $<0.001 *$ \\
\hline Broad-spectrum antibiotics, n (\%) & $137(50.4)$ & $123(62.1)$ & $40(78.4)$ & $<0.001 *$ \\
\hline CVC, n (\%) & $80(29.4)$ & $73(36.9)$ & $24(47.1)$ & $0.03 *$ \\
\hline Indwelling urinary catheter, n (\%) & $83(30.5)$ & $86(43.4)$ & $32(62.7)$ & $<0.001 *$ \\
\hline TPN, n (\%) & $8(2.9)$ & $6(3.0)$ & $5(9.8)$ & 0.05 \\
\hline Prolonged use of GC, n (\%) & $23(8.5)$ & $\mathrm{I}(0.5)$ & $0(0)$ & $<0.001 *$ \\
\hline Immunosuppressive therapy, $\mathrm{n}(\%)$ & $26(9.6)$ & II (5.6) & $3(5.9)$ & 0.24 \\
\hline \multicolumn{5}{|l|}{ Laboratory Data } \\
\hline Neutropenia, n (\%) & $12(4.4)$ & $7(3.5)$ & $\mathrm{I}(2.0)$ & 0.68 \\
\hline Grade I, n (\%) & $4(1.5)$ & $\mathrm{I}(0.5)$ & $0(0)$ & \\
\hline Grade II, n (\%) & $4(1.5)$ & $\mathrm{I}(0.5)$ & $0(0)$ & \\
\hline Grade III, n (\%) & $2(0.7)$ & $\mathrm{I}(0.5)$ & $\mathrm{I}(2.0)$ & \\
\hline Grade IV, n (\%) & I $(0.4)$ & $3(1.5)$ & $0(0)$ & \\
\hline Lymphopenia, n (\%) & $79(29.0)$ & $71(35.9)$ & $25(49.0)$ & $0.02 *$ \\
\hline Anemia, n (\%) & $88(32.4)$ & $80(40.4)$ & $26(51.0)$ & $0.02 *$ \\
\hline Mild, n (\%) & $50(18.4)$ & $33(16.7)$ & II (2I.6) & \\
\hline Moderate, n (\%) & $33(12.1)$ & $42(21.2)$ & $15(29.4)$ & \\
\hline Severe, n (\%) & $5(1.8)$ & $4(2.0)$ & $0(0)$ & \\
\hline Very severe, n (\%) & $0(0)$ & $\mathrm{I}(0.5)$ & $0(0)$ & \\
\hline Hypoalbuminemia, n (\%) & $114(41.9)$ & $101(51.0)$ & $28(54.9)$ & \\
\hline All-cause mortality, n (\%) & $55(20.2)$ & $60(30.3)$ & $20(39.2)$ & $0.004^{*}$ \\
\hline
\end{tabular}

Note: ${ }^{*} P<0.05$.

Abbreviations: BMI, body mass index; CAD, coronary artery disease; CKD, chronic kidney disease; COPD, chronic obstructive pulmonary disease; CTD, connective tissue disease; CVC, central venous catheter; DM, diabetes mellitus; GC, glucocorticoid; ICU, intensive care unit; IFD, invasive fungal disease; SD, standard deviation; TPN, total parenteral nutrition. 
No patient received stem cell transplant. Hospital-acquired IFD occurred in $300(57.6 \%)$ patients, among which 67 $(22.3 \%)$ patients developed IFD in intensive care unit (ICU), and $35(11.7 \%)$ patients were using ventilation before IFD onset.

\section{Characterizations of IFD}

A total of $236(45.3 \%)$ patients were infected with Candida spp., 149 (28.6\%) with Aspergillus spp., 38 (6.9\%) with Mucor spp., 24 (4.6\%) with Cryptococcus spp., and 6 (1.2\%) with P. marneffei, and $2(0.4 \%)$ with Rhizopus spp. Mixed fungal infection was diagnosed in 40 (7.7\%) patients. Fungi were found but not classified in 28 (5.4\%) patients.

The common infective sites of invasive candidiasis (IC) included abdominal cavity $(83 / 236,35.2 \%)$, bloodstream $(55 / 236,23.3 \%)$, esophagus $(31 / 236,13.1 \%)$, and urinary tract $(28 / 236,11.9 \%)$. Nearly $31.4 \%$ (74/236) of the patients had disseminated candidiasis. Serum 1,3-b-D-glucan level (G-test) was positive in 33 out of $59(55.9 \%)$ patients. Three cases were diagnosed by biopsy or surgery, including one involving the lung, one involving posterior peritoneum, and one in the stomach.

Cryptococcal infection presented as pulmonary cryptococcosis $(18 / 24,75.0 \%)$, cryptococcal meningitis $(2 / 24$, $8.3 \%$ ), and disseminated cryptococcosis (4/24, 16.7\%). Serum cryptococcal antigen $(\mathrm{CrAg})$ was positive in 11 out of 14 patients $(78.6 \%)$. Twelve patients with pulmonary cryptococcosis were diagnosed by lung biopsy.

Invasive aspergillosis (IA) mainly developed in the sinus $(74 / 149,49.7 \%)$ and lung $(65 / 149,43.6 \%)$. Two (2/ $149,1.3 \%$ ) patients had disseminated aspergillosis involving the lung and other organs. As for pulmonary aspergillosis, $62(62 / 67,92.5 \%)$ patients had invasive pulmonary aspergillosis, $4(4 / 67,6.0 \%)$ patients had chronic pulmonary aspergillosis, and 1 (1/67, 1.5\%) patient presented tracheobronchitis. Serum galactomannan (GM) test was performed in 48 patients and positive in 17 $(35.4 \%)$. The positive rate of the GM test was higher in bronchoalveolar lavage fluid (BALF) (11/16, 68.8\%). Eighty-eight patients were diagnosed with pathological evidence, among which 5 cases were diagnosed by transbronchial lung biopsy and 83 by surgery.

Mucormycosis usually involved the sinus (26/38, $68.4 \%)$, and lung (10/38, 26.3\%). Twenty-nine cases of mucormycosis were established on surgical pathology.

Infections with $P$. marneffe $i$ were diagnosed in the lung $(3 / 6,50.0 \%)$, bloodstream $(2 / 6,33.3 \%)$, and soft tissue $(1 / 6,16.7 \%)$.

\section{Distribution of Isolated Fungi}

Five hundred and five (96.9\%) patients were diagnosed with etiological evidence. A total of 521 strains of fungi were isolated or recovered mainly from abdominal fluid $(n=111)$, blood $(n=89)$, sputum $(n=60)$, urine $(n=52)$, esophageal brushing sample $(n=31)$, BALF $(n=15)$, bile $(n=13)$, pleural fluid $(n=9)$, and cerebrospinal fluid (CSF) $(n=4)$. The distribution of isolated fungi is shown in Table 3. C. albicans $(176 / 521,33.8 \%)$ and A. fumigatus $(46 / 521,8.8 \%)$ were the leading strains.

Among 89 cases of fungemia, $73(82.0 \%)$ cases were candidemia, $4(4.5 \%)$ cases were cryptococcemia, and 2 (2.2\%) cases were infected by P. marneffei. Ten (11.2\%) patients had multiple fungi grown in blood samples. Blood samples were obtained from peripheral venous $(75 / 89$, $84.3 \%)$ or CVC (14/89, 15.5\%).

Drug resistance analysis showed that $4.7 \%(5 / 106)$ of C. albicans strains were resistant to itraconazole, $2.3 \%$ (3/ $133)$ to fluconazole, and $1.5 \%(2 / 133)$ to voriconazole. Approximately $21.4 \%$ (6/28) of C. glabrata strains were resistant to itraconazole, and $3.3 \%(1 / 30)$ to voriconazole. C. tropicalis strains had the highest drug resistance rate to itraconazole $(11 / 33,33.3 \%)$. Nearly $21.1 \%$ (8/38) of C. tropicalis strains were resistant to fluconazole, $13.2 \%$ $(5 / 38)$ to voriconazole, and $3.6 \%(1 / 28)$ to amphotericin B.

\section{Secular Trends of IFD in the Elderly Population}

The annual prevalence of IFD ranged from $0.1 \%$ to $0.5 \%$ (Figure 1). The distribution of each IFD subtype was further analyzed. The proportion of candidiasis declined from $77.8 \%$ in year 1998 to $38.5 \%$ in year 2018. In contrast, aspergillosis consisted of increasing cases, and the proportion increased from $11.1 \%$ in year 1998 to $28.8 \%$ in year 2018 .

\section{Characteristics of IFD in Patients with Different Underlying Conditions}

Since the majority of the elderly patients had chronic conditions including DM, malignancy, COPD, CKD or received recent operation, we further analyzed the distribution of infective agents and infective sites in patients with different underlying conditions. As shown in Figure 2A, candidiasis was the most common infectious pattern in patients with DM (59/126, 46.8\%), solid organ malignancy $(84 / 114,73.7 \%)$, CKD $(40 / 62,64.5 \%)$ or receiving operation $(109 / 147,74.1 \%)$. Patients with COPD were likely to develop aspergillosis (25/51, 
Table 3 Distribution of Isolated Fungi

\begin{tabular}{|l|l|}
\hline Isolated Fungi & $\mathbf{N}(\%)$ \\
\hline Total & $\mathbf{5 2 I}$ \\
Candidaspp. & $292(56.0)$ \\
C. albicans & $176(33.8)$ \\
C. tropicalis & $41(7.9)$ \\
C. glabrata & $32(6.1)$ \\
C. parapsilosis & $32(6.1)$ \\
C. krusei & $3(0.6)$ \\
C. lusitaniae & $3(0.6)$ \\
C. guilliermondii & $2(0.4)$ \\
C. famata & $2(0.4)$ \\
C. lipolytica & $1(0.2)$ \\
\hline Aspergillusspp. & $157(30.1)$ \\
A. fumigatus & $46(8.8)$ \\
A. flavus & $15(2.9)$ \\
A. niger & $4(0.8)$ \\
A. terreus & $1(0.2)$ \\
Aspergillus unclassified & $92(17.7)$ \\
\hline Zygomycetesspp. & $45(8.6)$ \\
Mucor spp. & $43(8.3)$ \\
Rhizopus spp. & $2(0.4)$ \\
\hline Cryptococcusspp. & $20(3.8)$ \\
C. neoformans & $18(3.5)$ \\
C. laurentii & $2(0.4)$ \\
\hline Penicilliumspp. & $7(1.3)$ \\
P. marneffei & $7(1.3)$ \\
\hline & \\
\hline
\end{tabular}

49.0\%). In consistent, patients with COPD usually developed IFD in the lung $(32 / 51,62.7 \%)$, while abdominal cavity was commonly affected in patients with malignancy $(44 / 114,38.6 \%)$, CKD $(31 / 62,50.0 \%)$ or receiving operation $(62 / 147,42.2 \%)$ (Figure $2 \mathrm{~B})$. Of note, among 114 patients with solid organ malignancy, $63.2 \%(72 / 114)$ of them received abdominal operation. Among 147 patients who received operation, 76.9\% $(113 / 147)$ of them underwent abdominal operation. Approximately $46.8 \%(29 / 62)$ of the patients with CKD was on continuous ambulatory peritoneal dialysis (CAPD). IFD in other comorbidities was not analyzed given only a small number of patients were included.

\section{Age and IFD}

Patients were divided into three groups based on the age at admission. The distribution of IFD subtypes is shown in Figure 3A. Candidiasis (40.8\% in age 60-69, 47.0\% in age $70-79,62.7 \%$ in age $\geq 80)$ and aspergillosis (31.3\% in age $60-69,26.3 \%$ in age $70-79,23.5 \%$ in age $\geq 80$ ) constituted most of the infections irrespective of age. The proportion of mixed fungi infection was slightly increased in patients over 70 years of age $(5.1 \%$ in age $60-69,11.6 \%$ in age $70-79,5.9 \%$ in age $\geq 80$ ). The infective sites of IFD are summarized in Figure 3B. Most of the patients with age 60-69 developed IFD in the sinus $(78 / 272,28.7 \%)$, lung $(57 / 272,21.0 \%)$ or abdominal cavity $(53 / 272,19.5 \%)$. The proportion of disseminated infection increased remarkably in the oldest-old (age $\geq 80$ years) patient subset (17/ $51,33.3 \%)$.

\section{Treatment and Prognosis}

Treatment data were documented in $516(99.0 \%)$ patients. Nearly $64.0 \%(330 / 516)$ of the patients received antifungal medications, 24.2\% (125/516) received operation alone, and $2.5 \%(13 / 516)$ received operation and subsequent antifungal therapy. Thirty-three $(6.4 \%)$ cases of IFD remained untreated. The diagnosis of IFD was confirmed in $15(2.9 \%)$ patients after death. Presumptive antifungal treatment was given to $69(13.4 \%)$ patients. Nearly $69.4 \%$ $(358 / 516)$ of the patients received initial adequate antifungal treatment.

Voriconazole remains to be the primary treatment for aspergillosis during the past 20 years in our study. The proportion of voriconazole use ranged from $33.3 \%$ to $56.3 \%$. The use of echinocandins increased with time. During the year 2004-2008, 15.4\% of the patients were treated with echinocandins, while the number increased to $26.1 \%$ during the year 2014-2018. Treatment for mucormycosis and penicilliosis did not differ over time. Patients with mucormycosis received amphotericin B. Penicillosis were treated with itraconazole or voriconazole.

Fluconazole is the primary treatment for candidiasis. Nearly $94.4 \%$ of patients received fluconazole during the year 2004-2008. However, the number declined to $46.3 \%$ during the year 2014-2018. In contrast, the use of echinocandins increased from $0 \%$ in the year 2004-2008 to $37.4 \%$ in the year $2014-2018$.

Severe adverse events were reported in $3(0.9 \%)$ patients, including impaired kidney function due to amphotericin $B(n=1)$, mental symptom due to voriconazole $(n=1)$, and heart failure due to itraconazole $(n=1)$. Three deceased patients received empirical anti-fungal treatment with agents that were proved afterwards not sensitive to the pathogens.

The all-cause mortality rate was $25.9 \%$ (135/521), and the highest mortality was observed in patients aged over 80 


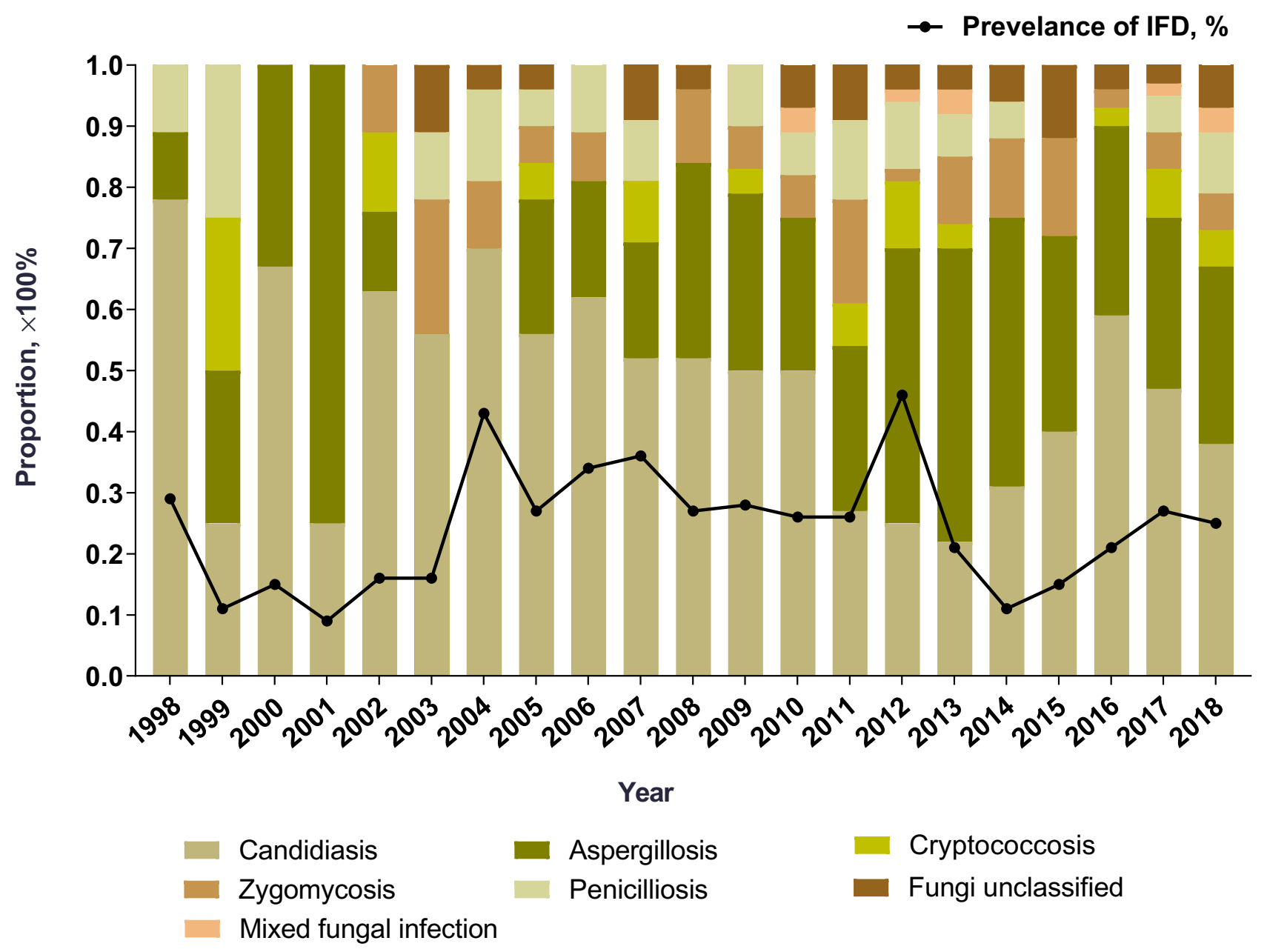

Figure I Prevalence of IFD and the distribution of IFD subtypes in elderly patients since 1998. Abbreviation: IFD, invasive fungal disease.

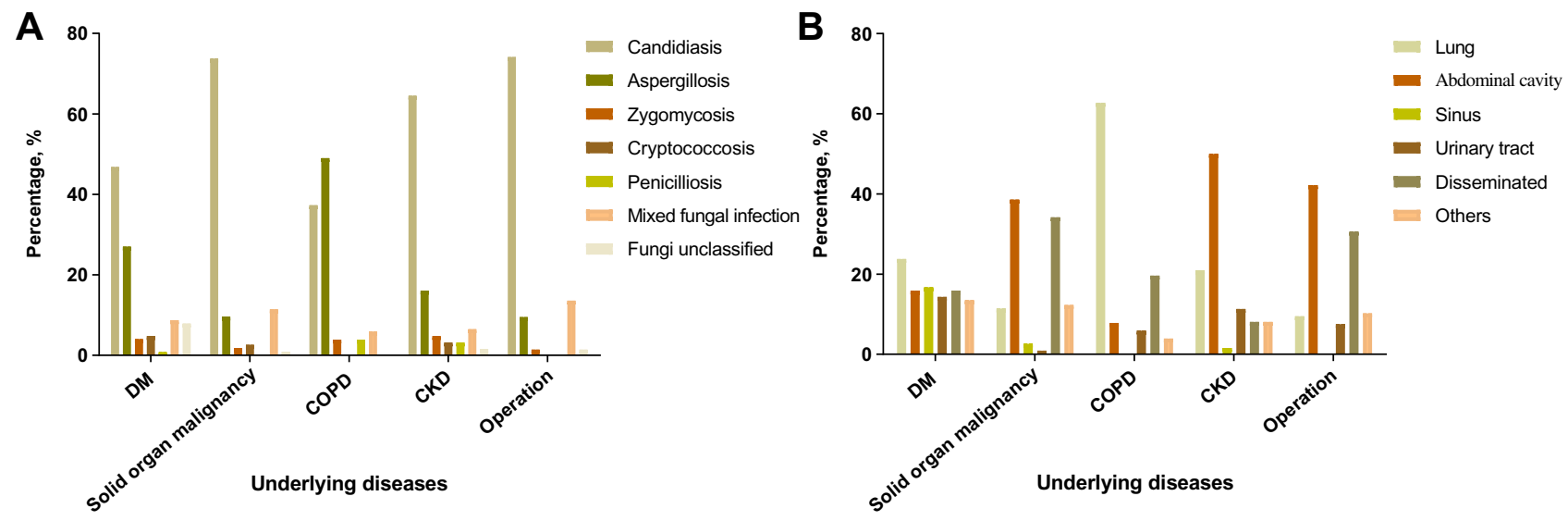

Figure 2 Infectious patterns and infective sites of IFD inelderly patients with different underlying diseases. (A) Infectious patterns; (B) Infective sites. Abbreviations: CKD, chronic kidney disease; COPD, chronic obstructive pulmonary disease; DM, diabetes mellitus; IFD, invasive fungal disease.

years $(20 / 51,39.2 \%)$. Seventy-six (56.3\%) deceased patients had candidiasis, and 29 (21.5\%) had aspergillosis. Mixed fungal infection was diagnosed in $13.3 \%(18 / 135)$ of the death cases. As for infective sites, disseminated fungal infection $(46 / 135,34.1 \%)$ was the most common, followed by lung infection $(38 / 135,28.1 \%)$. Based on the subtypes of 

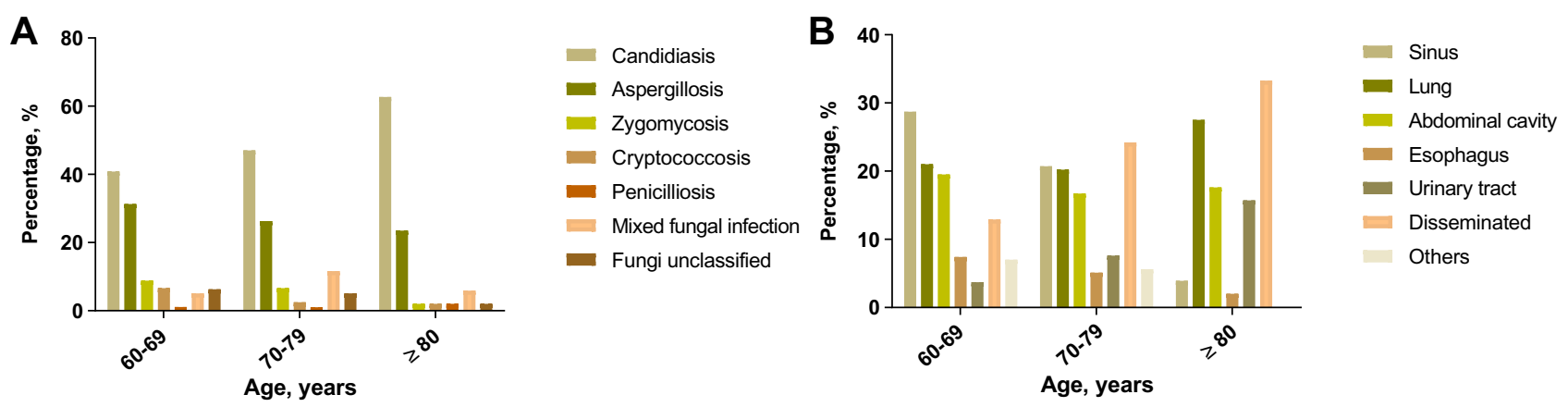

Figure 3 Infectious patterns (A) and infective sites (B) of IFD in elderly patients, by age. Abbreviation: IFD, invasive fungal disease.

IFD, mixed fungal infection $(18 / 40,45.0 \%)$ was the most fatal, followed by penicilliosis $(2 / 6,33.3 \%)$, candidiasis $(76 /$ $236,32.2 \%)$, aspergillosis $(29 / 149,19.5 \%)$, zygomycosis (6/ $38,15.8 \%)$, and cryptococcosis $(3 / 24,12.5 \%)$. One deceased patient was infected with unclassified fungus. Causes for death included septic shock $(83 / 135,61.5 \%)$, respiratory failure $(27 / 135,20.0 \%)$, cerebral hemorrhage $(8 / 135$, $5.9 \%)$, gastrointestinal hemorrhage $(7 / 135,5.2 \%)$, heart failure (4/135, 3.0\%), acute cardiac infarction (4/135, 3.0\%), massive hemoptysis $(1 / 135,0.7 \%)$, and disseminated intravascular coagulation (DIC) $(1 / 135,0.7 \%)$.

\section{Comparison Between Survivors and Non-Survivors with Invasive Mold Infection}

Since a majority of patients were infected with molds (ie, aspergillosis, mucormycosis, penicilliosis) or Candida spp., and these two types of fungi enter human body in different routes, we further conducted comparisons between survivors and non-survivors in different infection subtypes. As shown in Table 4, Charlson score was higher in non-survivors with mold infection $(5.1 \pm 1.9$ vs $3.3 \pm 1.6$, $P<0.001)$. More patients received $>10$ medications before IFD onset in the non-survivor group $(48.6 \%$ vs $4.5 \%$, $P<0.001)$. The deceased patients tended to complicate with malignancy $(24.3 \%$ vs $7.1 \%, P=0.002)$, DM (35.1\% vs $17.9 \%, P=0.02), \mathrm{CKD}(24.3 \%$ vs $3.8 \%, P<0.001)$, or COPD $(29.7 \%$ vs $11.5 \%, P=0.01)$. Non-survivors received more broad-spectrum antibiotics $(86.5 \%$ vs $26.3 \%$, $P<0.001)$, GC ( $13.5 \%$ vs $2.6 \%, P=0.01)$, or immunosuppressants $(13.5 \%$ vs $3.8 \%, P=0.02)$ prior to invasive mold infection. Neutropenia ( $10.8 \%$ vs $5.8 \%, P=0.02)$, lymphopenia $(59.5 \%$ vs $17.3 \%, P<0.001)$, anemia $(48.6 \%$ vs $17.3 \%, P<0.001)$ and hypoalbuminemia $(64.9 \%$ vs $27.6 \%, P<0.001)$ was significant in deceased patients.
Multivariate logistic regression analysis selected the following variables: age, tobacco, Charlson score, polypharmacy, hematological or solid organ malignancy, DM, $\mathrm{CKD}$, COPD, prolonged hospitalization, broad-spectrum antibiotics, prolonged use of GC, immunosuppressive therapy, neutropenia, lymphopenia, anemia, hypoalbuminemia, and initial adequate treatment, according to the clinical relevance and univariate analysis. Considering significant disproportion exists in ICU admission between groups, this factor was not included in multivariate regression analysis. Results showed that polypharmacy (adjusted OR 6.11, 95\% CI 2.03-18.36, $P=0.001$ ), CKD (adjusted OR $4.45,95 \%$ CI $1.18-16.73 P=0.03$ ), broad-spectrum antibiotics (adjusted OR 6.35, 95\% CI $1.96-20.60$ $P=0.002$ ) and lymphopenia (adjusted OR 3.02, 95\% CI $1.15-7.93, P=0.03]$ were associated with mortality in the elderly patients with invasive mold infection.

\section{Comparison Between Survivors and Non-Survivors with IC}

A comparison between survivors and non-survivors with IC (Table 5) showed that non-survivors were more likely to receive broad-spectrum antibiotics $(89.5 \%$ vs $70.6 \%$, $P=0.001)$, CVC (68.4\% vs $40.6 \%, P<0.001)$, and indwelling urinary catheter $(68.4 \%$ vs $46.3 \%, P=0.001)$. A higher proportion of disseminated candida infection was observed in non-survivors $(46.1 \%$ vs $24.4 \%, P=0.001)$. The distribution of neutropenia and lymphopenia did not differ between groups.

Factors with statistical significance in univariate analysis were considered clinically associated with IC, and thus included in multivariate regression analysis. Results showed that ICU admission (adjusted OR 7.58, 95\% CI $3.91-14.71, P<0.001)$ were associated with mortality in elderly patients with IC. 
Table 4 Comparison Between Survivors and Non-Survivors with Invasive Mold Infection

\begin{tabular}{|c|c|c|c|c|c|}
\hline & $\begin{array}{l}\text { Survivors } \\
(n=156)\end{array}$ & $\begin{array}{l}\text { Non-Survivors } \\
(n=37)\end{array}$ & $P$ value & Crude OR $(95 \% \mathrm{Cl})$ & $\begin{array}{l}\text { Adjusted OR } \\
(95 \% \mathrm{Cl})\end{array}$ \\
\hline \multicolumn{6}{|l|}{ Baseline Characteristics } \\
\hline Age, years, mean $\pm S D$ & $68.0 \pm 6.0$ & $67.5 \pm 8.5$ & $<0.00$ I* & $1.10(1.04-1.16)^{*}$ & \\
\hline Male:Female & $85: 71$ & $26: 11$ & 0.13 & & \\
\hline $\mathrm{BMI}, \mathrm{kg} / \mathrm{m}^{2}$, mean $\pm \mathrm{SD}$ & $23.1 \pm 3.2$ & $22.8 \pm 2.3$ & 0.58 & & \\
\hline Tobacco use, $\mathrm{n}(\%)$ & $32(20.5)$ & $14(37.8)$ & $0.03 *$ & $2.36(1.09-5.09)^{*}$ & \\
\hline Charlson score, mean $\pm \mathrm{SD}$ & $3.3 \pm 1.6$ & $5.1 \pm 1.9$ & $<0.001 *$ & $1.68(1.36-2.08)^{*}$ & \\
\hline Polypharmacy, n (\%) & $7(4.5)$ & $18(48.6)$ & $<0.001 *$ & $20.17(7.46-54.55)^{*}$ & $6.11(2.03-18.36)^{*}$ \\
\hline \multicolumn{6}{|l|}{ Comorbidities } \\
\hline Hypertension, n (\%) & $54(34.6)$ & $16(43.2)$ & 0.33 & & \\
\hline Hematological or solid organ malignancy, $n(\%)$ & $8(7.1)$ & $9(24.3)$ & $0.002 *$ & $3.64(1.35-9.83)^{*}$ & \\
\hline $\mathrm{DM}, \mathrm{n}(\%)$ & $28(17.9)$ & $13(35.1)$ & $0.02 *$ & $2.48(1.13-5.45)^{*}$ & \\
\hline CKD, n (\%) & $6(3.8)$ & $9(24.3)$ & $<0.001 *$ & $8.04(2.65-24.36)^{*}$ & $4.45(1.18-16.73)^{*}$ \\
\hline CTD, n (\%) & $6(3.8)$ & $4(10.8)$ & 0.09 & & \\
\hline CAD, n (\%) & $16(10.3)$ & $3(8.1)$ & 0.69 & & \\
\hline COPD, n (\%) & $18(11.5)$ & II (29.7) & $0.01 *$ & $3.24(1.37-7.66)^{*}$ & \\
\hline Solid organ transplantation, $\mathrm{n}(\%)$ & $I(0.6)$ & $\mathrm{I}(2.7)$ & 0.27 & & \\
\hline Acute stroke, $\mathrm{n}(\%)$ & $3(1.9)$ & I (2.7) & 0.76 & & \\
\hline \multicolumn{6}{|l|}{ Associated Factors } \\
\hline Prolonged hospitalization, n (\%) & $2(1.3)$ & $3(8.1)$ & $0.02 *$ & $6.79(1.09-42.24)^{*}$ & \\
\hline Recent operation, $\mathrm{n}(\%)$ & $5(3.2)$ & II (29.7) & $<0.001 *$ & $12.78(4.10-39.80)^{*}$ & \\
\hline ICU admission, $n(\%)$ & $14(9.0)$ & $34(91.9)$ & $<0.00 I^{*}$ & | $14.95(31.27-422.59)^{*}$ & \\
\hline Broad-spectrum antibiotics, n (\%) & $41(26.3)$ & $32(86.5)$ & $<0.001 *$ & $17.95(6.55-49.17)^{*}$ & $6.35(1.96-20.60)^{*}$ \\
\hline CVC, n (\%) & $11(7.1)$ & $24(64.9)$ & $<0.001 *$ & $24.34(9.76-60.56)^{*}$ & \\
\hline Indwelling urinary catheter, $\mathrm{n}(\%)$ & $15(9.6)$ & $30(81.1)$ & $<0.001 *$ & $40.29(15.12-107.33)^{*}$ & \\
\hline TPN, n (\%) & $3(1.9)$ & $4(10.8)$ & $0.01 *$ & $6.18(1.32-28.94)^{*}$ & \\
\hline Prolonged use of GC, $\mathrm{n}(\%)$ & $4(2.6)$ & $5(13.5)$ & $0.01^{*}$ & $5.94(1.51-23.34)^{*}$ & \\
\hline Immunosuppressive therapy, $\mathrm{n}(\%)$ & $6(3.8)$ & $5(13.5)$ & $0.02 *$ & $3.91(1.12-13.59)^{*}$ & \\
\hline Disseminated fungal infection, $n$ (\%) & $4(2.6)$ & I (2.7) & 0.96 & & \\
\hline \multicolumn{6}{|l|}{ Laboratory Data } \\
\hline Neutropenia, $\mathrm{n}(\%)$ & $4(5.8)$ & $4(10.8)$ & $0.02 *$ & $4.61(1.10-19.37)^{*}$ & \\
\hline Grade I, n (\%) & I (0.6) & $2(5.4)$ & & & \\
\hline Grade II, n (\%) & $2(1.3)$ & $0(0)$ & & & \\
\hline Grade III, n (\%) & $0(0)$ & I (2.7) & & & \\
\hline Grade IV, n (\%) & $I(0.6)$ & I (2.7) & & & \\
\hline Lymphopenia, n (\%) & $27(17.3)$ & $22(59.5)$ & $<0.001 *$ & $7.01(3.22-15.23)^{*}$ & $3.02(1.15-7.93)^{*}$ \\
\hline Anemia, $n(\%)$ & $27(17.3)$ & $18(48.6)$ & $<0.001 *$ & $4.53(2.10-9.74)^{*}$ & \\
\hline Mild, n (\%) & $17(10.9)$ & $6(16.2)$ & & & \\
\hline Moderate, $n(\%)$ & $9(5.8)$ & $12(32.4)$ & & & \\
\hline Severe, n (\%) & I $(0.6)$ & $0(0)$ & & & \\
\hline Very severe, $\mathrm{n}(\%)$ & $0(0)$ & $0(0)$ & & & \\
\hline Hypoalbuminemia, n (\%) & $43(27.6)$ & $24(64.9)$ & $<0.001 *$ & $4.85(2.27-10.38)^{*}$ & \\
\hline Initial adequate treatment, n (\%) & $137(87.8)$ & $24(64.9)$ & $0.001 *$ & $0.26(0.11-0.59)^{*}$ & \\
\hline
\end{tabular}

Note: $* p<0.05$

Abbreviations: BMI, body mass index; CAD, coronary artery disease; $\mathrm{Cl}$, confidential interval; CKD, chronic kidney disease; COPD, chronic obstructive pulmonary disease; CTD, connective tissue disease; CVC, central venous catheter; DM, diabetes mellitus; GC, glucocorticoid; ICU, intensive care unit; OR, odds ratio; SD, standard deviation; TPN, total parenteral nutrition.

\section{Discussion}

In this study, we demonstrated that candidiasis was the major IFD in the elderly patients, while aspergillosis constituted an increasing proportion. Patients at the extremes of age were particularly vulnerable to disseminated IFD. Pulmonary aspergillosis was likely to developed in patients with COPD, while IAC was common in patients receiving CAPD or gastrointestinal 
Table 5 Comparison Between Survivors and Non-Survivors with IC

\begin{tabular}{|c|c|c|c|c|c|}
\hline & $\begin{array}{l}\text { Survivors } \\
(n=160)\end{array}$ & $\begin{array}{l}\text { Non-Survivors } \\
(n=76)\end{array}$ & $P$ value & $\begin{array}{l}\text { Crude OR } \\
(95 \% \mathrm{Cl})\end{array}$ & $\begin{array}{l}\text { Adjusted OR } \\
(95 \% \mathrm{Cl})\end{array}$ \\
\hline \multicolumn{6}{|l|}{ Baseline Characteristics } \\
\hline Age, years, mean $\pm S D$ & $70.3 \pm 7.3$ & $70.9 \pm 10.8$ & 0.53 & $1.01(0.96-1.05)$ & \\
\hline Male:Female & 105:55 & $49: 27$ & 0.86 & & \\
\hline $\mathrm{BMI}, \mathrm{kg} / \mathrm{m}^{2}$, mean $\pm \mathrm{SD}$ & $21.5 \pm 3.3$ & $21.8 \pm 3.9$ & 0.56 & & \\
\hline Tobacco use, n (\%) & $44(27.5)$ & $12(15.8)$ & 0.05 & & \\
\hline Charlson score, mean $\pm S D$ & $5.0 \pm 2.1$ & $5.4 \pm 2.4$ & 0.72 & & \\
\hline Polypharmacy, n (\%) & $30(18.8)$ & $26(34.2)$ & $0.01 *$ & $2.25(1.21-4.18)^{*}$ & \\
\hline \multicolumn{6}{|l|}{ Comorbidities } \\
\hline Hypertension, n (\%) & $56(35.0)$ & $34(44.7)$ & 0.15 & & \\
\hline $\begin{array}{l}\text { Hematological or solid organ } \\
\text { malignancy, } \mathrm{n}(\%)\end{array}$ & $56(35.0)$ & $35(46.1)$ & 0.10 & $1.50(0.86-2.62)$ & \\
\hline DM, n (\%) & $41(25.6)$ & $18(23.7)$ & 0.75 & & \\
\hline CKD, n (\%) & $28(17.5)$ & $12(15.8)$ & 0.74 & & \\
\hline CTD, n (\%) & $13(8.1)$ & $\mathrm{I}(\mathrm{I} .3)$ & $0.04 *$ & $0.15(0.02-1.18)$ & \\
\hline CAD, n (\%) & $20(12.5)$ & II (I4.5) & 0.67 & & \\
\hline COPD, n (\%) & $14(8.8)$ & $5(6.6)$ & 0.57 & & \\
\hline Solid organ transplantation, $\mathrm{n}(\%)$ & I (0.6) & $4(5.3)$ & $0.02 *$ & $8.83(0.97-80.44)$ & \\
\hline Acute stroke, n (\%) & $4(2.5)$ & $3(3.9)$ & 0.54 & & \\
\hline \multicolumn{6}{|l|}{ Associated Factors } \\
\hline Prolonged hospitalization, n (\%) & $27(16.9)$ & $20(26.3)$ & 0.09 & $1.76(0.91-3.39)$ & \\
\hline Recent operation, n (\%) & $68(42.5)$ & $4 \mid(53.9)$ & 0.10 & $1.59(0.92-2.75)$ & \\
\hline ICU admission, $\mathrm{n}(\%)$ & $59(36.9)$ & $62(81.6)$ & $<0.00 I^{*}$ & $7.58(3.9 I-\mid 4.7 I) *$ & $7.58(3.9|-| 4.7 \mid)^{*}$ \\
\hline Broad-spectrum antibiotics, $\mathrm{n}(\%)$ & $113(70.6)$ & $68(89.5)$ & $0.001 *$ & $3.54(1.58-7.93)^{*}$ & \\
\hline CVC, n (\%) & $65(40.6)$ & $52(68.4)$ & $<0.00 I^{*}$ & $3.17(1.78-5.64)^{*}$ & \\
\hline Indwelling urinary catheter, n (\%) & $74(46.3)$ & $52(68.4)$ & $0.001 *$ & $2.52(1.42-4.47)^{*}$ & \\
\hline TPN, n (\%) & $5(3.1)$ & $4(5.3)$ & 0.42 & & \\
\hline Prolonged use of GC, n (\%) & $6(3.8)$ & $3(3.9)$ & 0.94 & & \\
\hline Immunosuppressive therapy, $\mathrm{n}(\%)$ & $12(7.5)$ & $8(10.5)$ & 0.44 & & \\
\hline Disseminated fungal infection, $\mathrm{n}(\%)$ & $39(24.4)$ & $35(46.1)$ & $0.001 *$ & $2.65(1.49-4.72)^{*}$ & \\
\hline \multicolumn{6}{|l|}{ Laboratory Data } \\
\hline Neutropenia, n (\%) & $5(3.1)$ & $3(3.9)$ & 0.74 & & \\
\hline Grade I, n (\%) & $\mathrm{I}(0.6)$ & $0(0)$ & & & \\
\hline Grade II, n (\%) & $\mathrm{I}(0.6)$ & $\mathrm{I}(\mathrm{I} .3)$ & & & \\
\hline Grade III, n (\%) & $2(1.3)$ & $\mathrm{I}(1.3)$ & & & \\
\hline Grade, IV, n (\%) & $\mathrm{I}(0.6)$ & $\mathrm{I}(\mathrm{I} .3)$ & & & \\
\hline Lymphopenia, n (\%) & $61(38.1)$ & $37(48.7)$ & 0.12 & & \\
\hline Anemia, n (\%) & $82(51.3)$ & $44(57.9)$ & 0.34 & & \\
\hline Mild, n (\%) & $37(23.1)$ & $21(27.6)$ & & & \\
\hline Moderate, n (\%) & $4 \mathrm{I}(25.6)$ & $19(25.0)$ & & & \\
\hline Severe, n (\%) & $4(2.5)$ & $3(3.9)$ & & & \\
\hline Very severe, n (\%) & $0(0)$ & $\mathrm{I}(\mathrm{I} .3)$ & & & \\
\hline Hypoalbuminemia, n (\%) & $89(55.6)$ & $5 I(67.1)$ & 0.09 & & \\
\hline Initial adequate treatment, $\mathrm{n}(\%)$ & $79(49.4)$ & $46(60.5)$ & 0.11 & & \\
\hline
\end{tabular}

Note: $* P<0.05$

Abbreviations: BMI, body mass index; CAD, coronary artery disease; $\mathrm{Cl}$, confidential interval; CKD, chronic kidney disease; COPD, chronic obstructive pulmonary disease; CTD, connective tissue disease; CVC, central venous catheter; DM, diabetes mellitus; GC, glucocorticoid; IC, invasive candidiasis; ICU, intensive care unit; OR, odds ratio; SD, standard deviation; TPN, total parenteral nutrition. 
operation. The mortality of IFD was high and increased with age.

In the general population, the incidences of IC and IA were stable during the last decade, and the overall incidence of IC was approximately 10 times higher than that of IA. ${ }^{18}$ In consistent, our results showed that the prevalence of IFD in elderly patients was stable during the last two decades, and IC remained to be the most common pattern, followed by IA. In the current study, the proportion of candidiasis tended to decline, while that of aspergillosis showed an upward trend in elderly people. One speculative reason is the advantage in diagnostic methods such as high-resolution computed tomography (HSCT) and GM test that improve the diagnosis accuracy of aspergillosis. Our finding suggests that increased vigilance against aspergillosis is necessary for timely diagnosis in elderly patients.

Patients with different underlying conditions are likely to be infected by different fungi. Generally, Candida spp. are common, except that in patients with COPD, Aspergillus spp. are more frequently found. Lung structure damage increases the risk of pulmonary infection due to the impairment of pathogens clearance. For example, monocyte-derived macrophages exhibit defective phagocytic and proinflammatory cytokine response to A. fumigatus, leading to fungal germination and dissemination in patients with COPD. ${ }^{21}$ Patients with DM are susceptible to candidiasis. Elevated blood glucose level creates a specific condition for intensive fungal colonization, ${ }^{22}$ and DM per se is an independent predictor for biofilm-forming Candida bloodstream infection. ${ }^{23}$ The proportions of candidiasis in patients with solid organ malignancy and CKD were extremely high in our study. One of the possible reasons is the performance of invasive procedure, including operation and CAPD. The alteration of intestinal flora is commonly found in elderly persons and is a potential risk factor for IAC. ${ }^{3}$ IAC was reported to occur in up to $40 \%$ of the patients after repeated gastrointestinal operation or acute abdomen. ${ }^{3}$ Therefore, the elderly patients with altered colonization of Candida strains are risky for candidiasis, especially in the situation of receiving abdominal operation or CAPD. Being aware of the different distributions of IFD in different comorbidities would guide the preference of antifungal medications.

An apparent disproportion of the infective patterns in patients from different age group was observed in our study. Patients of the oldest old are predisposed to disseminated infection. Many factors could contribute to this trend. Immune defense decreased gradually with age. $\mathrm{T}$ cells play a critical role in fungal defense. ${ }^{24,25}$ However, in the elderly with immunosenescence, skewing immune repertoire to the memory $\mathrm{T}$ cells is observed and the number of naïve $\mathrm{T}$ cells decreases due to thymus involution. $^{26}$ Age-related defect of IL-2 production impairs the expansion of $\mathrm{T}$ cells which is required for mounting the immune response. ${ }^{27}$ Besides, many risk factors for IFD such as the use of broad-spectrum antibiotics, indwelling catheterization, and lymphopenia were predominant in the oldest-old. Our results suggest that in patients with advanced age, active searching for potential infective sites of IFD is necessary.

During the last two decades, fluconazole and voriconazole remains to be the mainstay to treat candidiasis and aspergillosis, respectively. Meanwhile, we observed an upward trend in the use of echinocandins. Echinocandins are active against a broad spectrum of fungi, especially Candida spp., with few reported severe adverse effects and minimal drug-drug interactions. ${ }^{28}$ Therefore, echinocandins are encouraged to be used in elderly patients who usually take a high number of concomitant medicines. In contrast, only a small number of patients $(13 / 516,2.5 \%)$ in our study received amphotericin B (not amphotericin B liposome due to economic reasons). Considering the high incidence of nephrotoxicity of amphotericin B and the natural decline of renal function with advanced age, amphotericin B is not the first choice in the elderly populations. Besides, inpatients who were treated with highrisk drugs were closely monitored in our study. Once the adverse effects became severe, drugs were discontinued. In addition, we prescribed amphotericin B from a low dose and escalated gradually, following the dose-escalation strategy recommended in drug instruction in order to observe the infusion-related acute toxicity and doselimiting toxicity. These reasons could explain the difference in the incidence of adverse effects in our study and previous reports. ${ }^{29,30}$

The all-cause mortality in elderly patients with IFD is extremely high. In consistent with previous reports, ${ }^{9}$ neutropenia, prolonged use of GC and immunosuppressants were significant in non-survivors with invasive mold infection. More than half of the non-survivors had lymphopenia. Both $\mathrm{T}$ cells and $\mathrm{B}$ cells participate in hose defense against fungal infection. In particular, cytokines such as interferon (IFN)-c and tumor necrosis factor (TNF)- $\alpha$ released by T-helper type 1 (Th1) cells activate the innate immune system and stimulate B cells to produce 
fungi-specific antibodies. ${ }^{24,25}$ However, the number of active lymphocytes decreases with age physically, a fortiori, under the chronic conditions such as autoimmune disease or malignancy. Our results suggest that lymphopenia is an important predictor of mortality in invasive mold infection and needs special attention.

Candida spp. are the normal flora colonization over gastrointestinal or genitourinary tract. During aging process, metabolic disorders destroy the mucosal and cutaneous barrier through which the colonized Candida strains invade into deep-seated organs. ${ }^{18}$ In addition, old patients are more likely to receive invasive procedure during hospitalization such as urinary catheterization. Biofilm formation on the surface of the catheters is a critical problem and increases the difficulty for treatment. ${ }^{31}$ Reducing catheterization might reduce the risk of IC, otherwise, physicians should always bear in mind the possibility of IC in patients with indwelling catheters.

The strength of our study is the enrollment of a large number of patients from a long period of time. To the best of our knowledge, this study is the first to display the features of IFD in the elderly population from different angles, eg, time trend, comorbidities, and age. However, some limitations deserve comment. Firstly, data were generated from one hospital from Southern China, and only inpatients were included. The interpretation of the results to the general elderly population is cautious. Secondly, a small portion of invasive fungi were not classified due to technical or personal financial reasons, which could influence the prevalence of each IFD subtype. Thirdly, the retrospective nature has limited value in studying long-term prognosis. Initial adequate antifungal treatment is suggested to be critical to improve prognosis of IC. ${ }^{3}$ In our study, however, no advantage of initial adequate treatment was observed in patients who survived. One of the possible reasons could be that only initial treatment was evaluated in this study. Drug switching strategy and the course of treatment were not unified, and this could exert negative effect on treatment efficacy.

\section{Conclusion}

This study provides an overview of IFD in elderly patients. Candidiasis and aspergillosis were the major patterns. The mortality of IFD was high and increased with age. Increasing vigilance on lymphopenia, prompt appropriate clinical management and reduce invasive procedures could benefit the prognosis of IFD in subjects at risk. IFD is a great threat to the elderly. In-depth study on this issue will increase our knowledge of IFD management and benefit the prognosis in elderly population.

\section{Abbreviations}

ANC, absolute neutrophil count; BALF, bronchoalveolar lavage fluid; CAPD, continuous ambulatory peritoneal dialysis; CI, confidential interval; CKD, chronic kidney disease; COPD, chronic obstructive pulmonary disease; $\mathrm{CrAg}$, cryptococcal antigen; CSF, cerebrospinal fluid; CVC, central venous catheter; DIC, disseminated intravascular coagulation; DM, diabetes mellitus; GC, glucocorticoid; GM, galactomannan; HSCT, high-resolution computed tomography; IA, invasive aspergillosis; IAC, intraabdominal candidiasis; IC, invasive candidiasis; ICD-10, International Classification of Diseases; ICU, intensive care unit; IFD, invasive fungal disease; IFN, interferon; OR, odds ratio; $\mathrm{SD}$, standard deviation; TPN, total parenteral nutrition.

\section{Ethics Approval and Informed Consent}

Ethics committee of the First Affiliated Hospital of Sun Yat-sen University approved the research (Approval number: 2019422), waiving written informed consent for deidentified patient data.

\section{Data Sharing Statement}

The datasets generated and analyzed in the current study are available from the corresponding author on reasonable request.

\section{Author Contributions}

All authors contributed to data analysis, drafting or revising the article, gave final approval of the version to be published, and agree to be accountable for all aspects of the work.

\section{Funding}

This project was supported by the grants from the National Natural Science Foundation of China (81601403 to Minxi Lao, 81603435 to Dongying Chen).

\section{Disclosure}

The authors report no conflicts of interest in this work.

\section{References}

1. Dwyer LL, Harris-Kojetin LD, Valverde RH, et al. Infections in long-term care populations in the United States. $J$ Am Geriatr Soc. 2013;61(3):342-349. doi:10.1111/jgs.2013.61.issue-3 
2. Montecino-Rodriguez E, Berent-Maoz B, Dorshkind K. Causes, consequences, and reversal of immune system aging. J Clin Invest. 2013;123(3):958-965. doi:10.1172/JCI64096

3. Bassetti M, Righi E, Ansaldi F, et al. A multicenter multinational study of abdominal candidiasis: epidemiology, outcomes and predictors of mortality. Intensive Care Med. 2015;41(9):1601-1610. doi:10.1007/s00134-015-3866-2

4. Ramos-Martínez A, Vicente-López N, Sánchez-Romero I, et al. Epidemiology and prognosis of candidaemia in elderly patients Mycoses. 2017;60(12):808-817. doi:10.1111/myc.2017.60.issue-12

5. Martin GS, Mannino DM, Eaton S, Moss M. The epidemiology of sepsis in the United States from 1979 through 2000. N Engl J Med. 2003;348(16):1546-1554. doi:10.1056/NEJMoa022139

6. Li Y, Fang W, Jiang W, et al. Cryptococcosis in patients with diabetes mellitus II in mainland China: 1993-2015. Mycoses. 2017;60 (11):706-713. doi:10.1111/myc.2017.60.issue-11

7. Roden MM, Zaoutis TE, Buchanan WL, et al. Epidemiology and outcome of zygomycosis: a review of 929 reported cases. Clin Infect Dis. 2005;41(5):634-653.

8. Ascioglu S, Rex JH, de Pauw B, et al. Defining opportunistic invasive fungal infections in immunocompromised patients with cancer and hematopoietic stem cell transplants: an international consensus. Clin Infect Dis. 2002;34(1):7-14. doi:10.1086/323335

9. De Pauw B, Walsh TJ, Donnelly JP, et al. Revised definitions of invasive fungal disease from the European Organization for Research and Treatment of Cancer/Invasive Fungal Infections Cooperative Group and the National Institute of Allergy and Infectious Diseases Mycoses. Clin Infect Dis. 2008;46(12):1813-1821. doi:10.1086/588660

10. Scientific working group. The diagnosis and treatment of Candidiasis: the expert consensus. Clin J Infect Chemother. 2011;11 (2):81-95. Chinese

11. Kidney Disease: Improving Global Outcomes (KDIGO) CKD Work Group. KDIGO 2012 Clinical practice guideline for the evaluation and management of chronic kidney disease. Kidney Int Suppl. 2013;3 (1): $1-150$.

12. Joint Committee for Guideline Revision. 2018 Chinese guidelines for prevention and treatment of Hypertension-A report of the revision committee of chinese guidelines for prevention and treatment of hypertension. J Geriatr Cardiol. 2019;16(3):182-241. doi:10.11909/ j.issn.1671-5411.2019.03.014

13. Global initiative for chronic Obstructive Lung Disease. Global strategy for the diagnosis, management and prevention of COPD, 2018. Available from: https://www.goldcopd.org/. Accessed July 17, 2018.

14. Vogelmeier CF, Criner GJ, Martinez FJ, et al. Global strategy for the diagnosis, management, and prevention of chronic obstructive lung disease 2017 report. GOLD executive summary. Am J Respir Crit Care Med. 2017;195(5):557-582. doi:10.1164/rccm.201701-0218PP

15. Chinese Medical Association. Chinese guideline for type 2 diabetes mellitus. Chin J Diabetes. 2014;22(8):2-42. Chinese

16. Charlson ME, Pompei P, Ales KL, MacKenzie CR. A new method of classifying prognostic comorbidity in longitudinal studies: development and validation. J Chronic Dis. 1987;40(5):373-383. doi:10. 1016/0021-9681(87)90171-8
17. Patterson TF, Thompson GR 3rd, Denning DW, et al. Practice guidelines for the diagnosis and management of aspergillosis: 2016 update by the Infectious Diseases Society of America. Clin Infect Dis. 2016;63(4):e1-e60. doi:10.1093/cid/ciw326

18. Pfaller MA, Diekema DJ. Epidemiology of invasive candidiasis: a persistent public health problem. Clin Microbiol Rev. 2007;20 (1):133-163. doi:10.1128/CMR.00029-06

19. National Cancer Institute. Common terminology criteria for adverse events version 3.0. Available from: https://ctep.cancer.gov/protocolde velopment/electronic_applications/docs/ctcaev3.pdf. Accessed March 31, 2003.

20. Zhang ZN, Shen T. Diagnosis and Treatment of Blood Diseases. 3nd ed. Beijing: Science Press; 2007. Chinese.

21. Wrench C, Belchamber KBR, Bercusson A, et al. Reduced clearance of fungal spores by chronic obstructive pulmonary disease GM-CSFand M-CSF-derived macrophages. Am J Respir Cell Mol Biol. 2018;58(2):271-273. doi:10.1165/rcmb.2017-0351LE

22. Gosiewski T, Salamon D, Szopa M, et al. Quantitative evaluation of fungi of the genus Candida in the feces of adult patients with type 1 and 2 diabetes-A pilot study. Gut Pathog. 2014;6(1):43. doi:10.1186/ s13099-014-0043-z

23. Tumbarello M, Fiori B, Trecarichi EM, et al. Risk factors and outcomes of candidemia caused by biofilm-forming isolates in a tertiary care hospital. PLoS One. 2012;7(3):e33705. doi:10.1371/journal. pone.0033705

24. Wuthrich M, Deepe GS, Klein B. Adaptive immunity to fungi. Annu Rev Immunol. 2012;30:115-148. doi:10.1146/annurev-immunol -020711-074958

25. Leibundgut-landmann S, Wothrich M, Hohl TM. Immunity to fungi. Curr Opin Immunol. 2012;24(4):449-458. doi:10.1016/j.coi.2012. 04.007

26. Webster RG. Immunity to influenza in the elderly. Vaccine. 2000;18 (16):1686-1689. doi:10.1016/S0264-410X(99)00507-1

27. Nagel JE, Chopra RK, Chrest FJ, et al. Decreased proliferation, interleukin 2 synthesis, and interleukin 2 receptor expression are accompanied by decreased mRNA expression in phytohemagglutinin-stimulated cells from elderly donors. J Clin Invest. 1988;81(4):1096-1102. doi:10.1172/JCI113422

28. Andes DR, Safdar N, Baddley JW, et al. Impact of treatment strategy on outcomes in patients with candidemia and other forms of invasive candidiasis: a patient-level quantitative review of randomized trials. Clin Infect Dis. 2012;54(8):1110-1122. doi:10.1093/cid/cis021

29. Girois SB, Chapuis F, Decullier E, Revol BG. Adverse effects of antifungal therapies in invasive fungal infections: review and meta-analysis. Eur J Clin Microbiol Infect Dis. 2006;25(2):13 8-149. doi:10.1007/s10096-005-0080-0

30. Scott LJ. Micafungin: a review of its use in the prophylaxis and treatment of invasive Candida infections. Drugs. 2012;72 (16):2141-2165. doi:10.2165/11209970-000000000-00000

31. Douglas LJ. Candida biofilms and their role in infection. Trends Microbiol. 2003;11(1):30-36. doi:10.1016/S0966-842X(02)00002-1
Infection and Drug Resistance

\section{Publish your work in this journal}

Infection and Drug Resistance is an international, peer-reviewed openaccess journal that focuses on the optimal treatment of infection (bacterial, fungal and viral) and the development and institution of preventive strategies to minimize the development and spread of resistance. The journal is specifically concerned with the epidemiology of antibiotic resistance and the mechanisms of resistance development and diffusion in both hospitals and the community. The manuscript management system is completely online and includes a very quick and fair peerreview system, which is all easy to use. Visit http://www.dovepress.com/ testimonials.php to read real quotes from published authors. 